\title{
Integrals in Propagation of Uncertainty
}

Giorgio Gambirasio,giga@uol.com.br

\begin{abstract}
An alternative method to the one proposed by the Bureau International des Poids et Mesures - BIPM is investigated. Instead of using derivatives, integration is used to compute mean value and variance of measurands. Different cases are examined: linear electrical circuits, use of sigmoids, implicit functions

The integral method does not require the functions to be differentiable and is capable to perform well even when a function has a "jump" only describable with the help of a step-function.
\end{abstract}

Keywords: propagation of uncertainty, Bureau International des Poids et Mesures, BIPM, differentiation, integration, mean, variance, random variables, implicit functions, unit step function, sigmoid, Taylor series.

\section{Introduction}

It is a curious thing that at times certain areas of knowledge do not excite the interest of researchers. In a smaller scale, the case exposed in this paper belongs to such a category. Wikipedia defines "Propagation of Uncertainty " as the effect of variables' uncertainties (or errors, more specifically random errors) on the uncertainty of a function based on them. When the variables are the values of experimental measurements they have uncertainties due to measurement limitations (e.g., instrument precision) which propagate due to the combination of variables in the function.

The traditional method to handle the question starts by observing that the total differential $\mathrm{d} f$ of a function $f\left(\mathrm{x}_{1}, \mathrm{x}_{2}, \ldots, \mathrm{x}_{\mathrm{n}}\right)$ of $\mathrm{n}$ variables is given by $\mathrm{d} f=\sum_{i=1}^{n}\left(\frac{\partial f}{\partial x_{i}}\right) \cdot d x_{i}$. Or, alternatively, that $f$ can be approximated by the first terms of a Taylor series. But, after such declarations, the discourse is suddenly changed: variables $x_{i}$ are considered random variables (r.v.); function $f$ itself becomes a random variable; differentials $\mathrm{dx}_{\mathrm{i}}$ are equaled to the standard deviation $\sigma_{\imath}$ of the corresponding r.v. $x_{i}$. Finally, a formula from Statistics is applied to get the variance $\sigma_{\mathrm{f}}^{2}$ of the (now, a r.v.) $f\left(\mathrm{x}_{1}, \mathrm{x}_{2}, \ldots, \mathrm{x}_{\mathrm{n}}\right)$ :

$$
\sigma_{\mathrm{f}}^{2}=\sum_{i=1}^{n}\left(\frac{\partial f}{\partial x_{i}}\right)^{2} \cdot \sigma_{\mathrm{xi}}^{2}
$$

The formula represents the standard method to compute the propagation of uncertainty, as recommended by the Bureau International des Poids et Mesures (BIPM).

The BIPM is a modern spin-off of the French revolution that takes care, in an international basis, of all questions regarding measurement, standards, units of SI and related matters. The Bureau's page on the Internet (www.bipm.org) informs about its geographical address: approximately at latitude $48^{\circ}$ North and longitude $2^{\circ}$ East. This means Sèvres, near Paris. 
The formulas proposed by BIPM obviously require that function $f$ be differentiable with respect to all its variables. We will occasionally refer to IBPM's standard procedure as a "differential method" in contrast to an "integral method" as proposed in this paper.

It looks strange that such things as variance, quantity originally defined by integrals, should be computed by differentiation. Let us first consider the equations defining the mean $\mathrm{m}_{\mathrm{f}}$ and the variance $\sigma_{\mathrm{f}}^{2}$ of a function, itself a r.v. $f\left(\mathrm{x}_{1}, \mathrm{x}_{2}, \ldots, \mathrm{x}_{\mathrm{n}}\right)$, where the independent r.v. have the respective probability densities $\mathrm{g}_{1}\left(\mathrm{x}_{1}\right), \mathrm{g}_{2}\left(\mathrm{x}_{2}\right), \ldots, \mathrm{g}_{\mathrm{n}}\left(\mathrm{x}_{\mathrm{n}}\right)$. Since the variables are considered independent, the joint probability density $\mathrm{g}_{\mathrm{f}}$ of $f$ is given by the product of the individual densities:

$$
\mathrm{g}_{\mathrm{f}}\left(\mathrm{x}_{1}, \mathrm{x}_{2}, \ldots, \mathrm{x}_{\mathrm{n}}\right)=\mathrm{g}_{1}\left(\mathrm{x}_{1}\right), \mathrm{g}_{2}\left(\mathrm{x}_{2}\right) \ldots \mathrm{g}_{\mathrm{n}}\left(\mathrm{x}_{\mathrm{n}}\right) .
$$

Then the mean of $f$ is defined by:

$$
\mathrm{m}_{\mathrm{f}}=\iint \ldots \int f\left(\mathrm{x}_{1}, \mathrm{x}_{2}, \ldots, \mathrm{x}_{\mathrm{n}}\right) \cdot \mathrm{g}_{1}\left(\mathrm{x}_{1}\right) \cdot \mathrm{g}_{2}\left(\mathrm{x}_{2}\right) \ldots \mathrm{g}_{\mathrm{n}}\left(\mathrm{x}_{\mathrm{n}}\right) \cdot \mathrm{dx}_{1} \cdot \mathrm{dx} \mathrm{x}_{2} \ldots \mathrm{d} \mathrm{x}_{\mathrm{n}}
$$

where the limits of the $\mathrm{n}$ integrals are determined by the intervals where the respective densities are not zero (forming the hyper-volume of interest). In an abbreviated form, it can be written as

$$
\mathrm{m}_{\mathrm{f}}=\int \mathrm{f} \cdot \mathrm{g}_{\mathrm{f} \cdot \mathrm{dx}},
$$

where $d x=d x_{1} . d x_{2} \ldots d x_{n}$, and $\int$ represents the n-tuple integration.

The variance of $f$ is defined, also in abbreviated form, by:

$$
\sigma_{\mathrm{f}}^{2}=\int\left(f-m_{f}\right)^{2} \cdot g_{f} \cdot d x
$$

Being curious myself, I decided to study a little whether the direct use of the definitions of mean and variance could be a valid alternative to the standard methodology by BIPM. What if we replace differentiation by integration in the evaluation of uncertainty propagation? Who knows whether the exigence of differentiability could be eased, since integration is notoriously more tolerant with respect to a function's behavior?

So, this paper was written.

$* * * * * * * * * * * * * * * * * * * * * * * * * * *$

After this Introduction, in the next section a very simple electrical circuit (one battery, two resistors one of them a r.v.) is used to show the basic aspects of the two approaches (differential and integral). In the third section, a less simple electrical circuit helps in showing how to deal with three independent r.v.

The attention is then brought to the question of differentiability. As an auxiliary object of study, a water-heating system is chosen in which one of the r.v. is a resistor that is switched on/off by the flow of water. The purpose of the exercise is to see how the integral approach deals with this case of non-differential variable.

As a preliminary piece of information, the section on the water-heating system is preceded by a section on step functions and their simulators, the sigmoids.

The case of implicit functions is also covered to see how the integral method deals with this kind of functions. 


\section{A very simple case}

The ideas about using integration in place of differentiation for the purpose of evaluating the propagation of uncertainty will be exposed here with the help of a very simple electrical circuit: a battery, two resistors. Figure 1 shows the circuit.

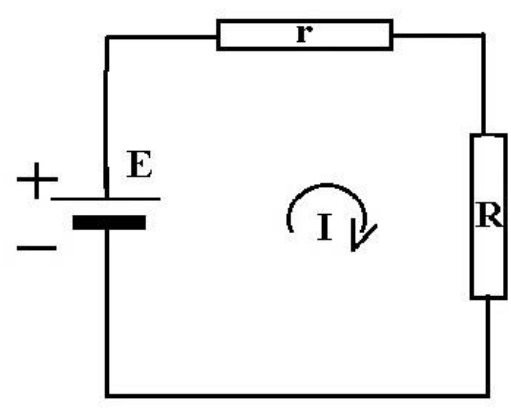

Fig. 1 - Battery E supplies the circuit with $1 \mathrm{~V}$; resistor $\mathrm{r}=1 \Omega$ is fixed; resistor $R=10 \pm 1 \Omega$ is a r.v.; current I is a r.v. function of R.

It happens that, for some reason, we do not exactly know the resistance of resistor R, but we do have some partial information. The reasons for our ignorance may be: incertitude from measurement, manufacturing differences, sensitivity to atmospheric conditions (temperature, humidity), and other. In account of such ignorance, we shall consider the resistance values as random variables (r.v.) and make use of the theory of random variables as the appropriate mathematical tool.

Resistor $\mathrm{r}$, on the contrary, is considered of fixed value, of $1 \mathrm{ohm}$, then not a r.v.

So far, the approach is the same as the one suggested by BIPM. The difference stays in the choice of tools: BIPM prefers differentiation, but now we explore the forgotten paths of integration.

Current I in the circuit also becomes a r.v., in the sense that it is a function of another r.v.: $\mathrm{I}=\mathrm{E} /(\mathrm{r}+\mathrm{R})$. Battery voltage is considered fixed, not a r.v., with voltage E equal to 1 volt. This circuit with one r.v. only will be sufficient to introduce the reader to the integral method and to compare its results with the method of differentiation. In the next section a case with more r.v. will be considered.

What we know about our random variables? We know some properties like mean or nominal values, variance or limits of variation. There certainly is a probability density function associated with each variable, but we do not know it. Since densities must be known to rightly perform the integrations, for simplicity densities are usually assumed to be uniform between given limits, and equal to zero outside such limits.

In this example, r.v. $\mathrm{R}$ is represented by a resistor of value equal to $10 \pm 1 \Omega$. Variables that have uniform probability density function $f=(1 /(b-a))$ over the interval $(a, b)$ are considered to have a variance $\sigma^{2}=(b-a)^{2} / 12$, then $\sigma_{R}{ }^{2}=0.333 \Omega^{2}$ and $\sigma_{R}=0.5773 \Omega$. 
The probability density is $f_{R}=0.5 \Omega^{-1}$ for $R$ over the interval $(9,11)$, and $f_{R}=0$ for $R$ out of the interval.

In our presentation we will rather loosely mix the r.v. $\mathrm{R}$ and its associated variable $\mathrm{x}$ in the formulas. For example, we may write expressions like:

$$
\begin{gathered}
\mathrm{I}=\mathrm{E} /(\mathrm{r}+\mathrm{R})=\mathrm{E} /(\mathrm{r}+\mathrm{x}), \\
\mathrm{dI} / \mathrm{dR}=(\mathrm{d} / \mathrm{dR})[\mathrm{E} /(\mathrm{r}+\mathrm{R})]=(\mathrm{d} / \mathrm{dx})[\mathrm{E} /(\mathrm{r}+\mathrm{x})] .
\end{gathered}
$$

This behavior is not recommended by mathematicians but is practical for and practiced by engineers. In the document "Evaluation of measurement data - Guide to the expression of uncertainty in measurement", produced by the Joint Committee for Guides in Metrology of BIPM, it is stated: For economy of notation, in this Guide the same symbol is used for the physical quantity (the measurand) and for the random variable that represents the possible outcome of an observation of that quantity.

Let us start with the BIPM approach. The nominal value of I is immediately computed:

$$
\mathrm{I}=\mathrm{E} /(\mathrm{r}+\mathrm{R})=1 /(1+10)=0.0909091 \mathrm{~A} .
$$

The variance $\sigma_{\mathrm{I}}^{2}$, in this particular case of only one r.v., is given by $\sigma_{\mathrm{I}}^{2}=(\mathrm{dI} / \mathrm{dR})^{2} \cdot \sigma_{\mathrm{R}}^{2}$, where the derivative is to be numerically computed for the nominal value of $\mathrm{R}$ :

$$
\begin{gathered}
\mathrm{dI} / \mathrm{dR}=(\mathrm{d} / \mathrm{dx})[\mathrm{E} /(\mathrm{r}+\mathrm{x})]=-1 /\left.(\mathrm{r}+\mathrm{x})^{2}\right|_{\mathrm{x}=\mathrm{R}}=-1 /(1+10)^{2} \\
\sigma_{\mathrm{I}}^{2}=(\mathrm{dI} / \mathrm{dR})^{2} \cdot \sigma_{\mathrm{R}}^{2}=(1 / 11)^{4} \cdot(0.333)=0.0000227 \mathrm{~A}^{2} ; \\
\sigma_{\mathrm{I}}=0.004771 \mathrm{~A} .
\end{gathered}
$$

It is usual to express the result as "(nominal value) \pm (standard deviation)". Then, the value $\mathrm{I}=0.0910 \pm 0.0048 \mathrm{~A}$ may be attributed to the current.

Next, let us do the analysis by integration.

The mean $\mathrm{m}_{\mathrm{I}}$ of the current is given by its defining expression:

$$
\mathrm{m}_{\mathrm{I}=} \int[1 /(\mathrm{r}+\mathrm{x})] \cdot \mathrm{f}_{\mathrm{R}}(\mathrm{x}) \cdot \mathrm{dx}=(0 \cdot 5) \cdot \int_{9}^{11}\left[\frac{1}{1+x}\right] \cdot d x=0.09116 \mathrm{~A} .
$$

This is almost equal to the nominal value.

Why is it not identical with the nominal value? Perhaps, because of numerical approximations in the integral computation? No, but because the definition of $\mathrm{m}_{\mathrm{I}}$ is different from the definition of nominal value. The latter is fixed; the former is a function of the probability density. For example, in the generic case r.v. $R=10 \pm z$ ohms, then

$$
\mathrm{m}_{\mathrm{I}}=\left(1 /(2 \mathrm{z}) \int_{10-z}^{10+z}\left[\frac{1}{1+x}\right] \cdot d x=(1 /(2 \mathrm{z}) \cdot \ln [(11+\mathrm{z}) /(11-\mathrm{z})] .\right.
$$


Mean $\mathrm{m}_{\mathrm{I}}$ may go from $\mathrm{m}_{\mathrm{I}}=\mathrm{I}=0.0909091$ (when $\mathrm{z}=0$, that is, $\mathrm{R}$ is fixed), to some value as high as $\mathrm{m}_{\mathrm{I}}=0.095$ when $\mathrm{R}$ approaches $\mathrm{R}=10 \pm 40 \%$.

Similar considerations may be done whenever values obtained from the two methods (differential, integral) are compared.

The variance $\sigma_{I}^{2}$ of the current is also computed by the definition:

$$
\begin{gathered}
\sigma_{\mathrm{I}}^{2}=\int\left[(1 /(\mathrm{r}+\mathrm{x}))-\mathrm{m}_{\mathrm{I}}\right]^{2} \cdot \mathrm{f}_{\mathrm{R}}(\mathrm{x}) \cdot \mathrm{dx}= \\
=(0.5) \cdot \int_{9}^{11}\left[\frac{1}{1+x}-0.09116\right]^{2} \cdot d x=0.0000230458 \mathrm{~A} .
\end{gathered}
$$

The standard deviation is $\sigma_{\mathrm{I}}=0.00480 \mathrm{~A}$. Then the current $\mathrm{I}=0.0912 \pm 0.0048 \mathrm{~A}$, similar to the value obtained with the differential approach.

\section{A typical electrical circuit}

A very simple electrical circuit was used in the preceding section just to give a first idea about how the two methods (derivation and integration) work for studying uncertainty propagation. There was only one r.v., which only required one integral.

In this section we shall consider a more realistic electrical circuit, with three r.v. therefore requiring a triple integration.

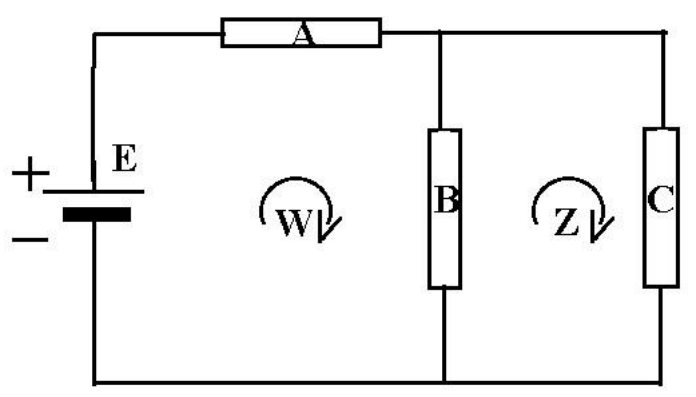

Fig, 2 - Electrical circuit that includes three independent random variables: resistors $\mathrm{A}, \mathrm{B}, \mathrm{C}$. The statistical properties of current $\mathrm{W}$, a r.v. function of $\mathrm{A}$, $\mathrm{B}, \mathrm{C}$, are calculated. Numerical values of components are shown in Table I. 
Table I - Components Characteristics

\begin{tabular}{|c|c|c|c|c|}
\hline \begin{tabular}{|c|} 
component// \\
symbol
\end{tabular} & nominal value & $\begin{array}{c}\text { dummy } \\
\text { variable }\end{array}$ & variance & $\begin{array}{c}\text { probability } \\
\text { density }\end{array}$ \\
\hline battery $\mathrm{E}$ & $1 \mathrm{~V}$, fixed & & & \\
\hline resistor A & $2 \pm 0.2 \Omega$ & $\mathrm{x}$ & $\begin{array}{c}\sigma_{\mathrm{A}}^{2}= \\
=(0.4)^{2} / 12= \\
=0.01333 \Omega^{2}\end{array}$ & $\begin{aligned} & \mathrm{f}_{\mathrm{A}}(\mathrm{x})= \\
= & 1 / 0.4 \Omega^{-1}\end{aligned}$ \\
\hline resistor B & $3 \pm 0.3 \Omega$ & $\mathrm{y}$ & $\begin{array}{c}\sigma_{\mathrm{B}}^{2}= \\
=(0.6)^{2} / 12= \\
=0.03 \Omega^{2}\end{array}$ & $\begin{aligned} & f_{B}(y)= \\
= & 1 / 0.6 \Omega^{-1}\end{aligned}$ \\
\hline resistor $\mathrm{C}$ & $4 \pm 0.4 \Omega$ & $\mathrm{z}$ & $\begin{array}{c}\sigma_{\mathrm{C}}^{2}= \\
=(0.8)^{2} / 12= \\
=0.0533 \Omega^{2}\end{array}$ & $\begin{array}{r}\mathrm{f}_{\mathrm{C}}(\mathrm{z})= \\
=1 / 0.8 \Omega^{-1}\end{array}$ \\
\hline current W & $\begin{array}{c}\text { E. }(B+C) /(A \cdot B+B . C+C . A)= \\
\quad=7 / 26=0.26923 \mathrm{~A}\end{array}$ & & & \\
\hline current Z & $\begin{array}{l}\text { E.B } /(A \cdot B+B \cdot C+C \cdot A)= \\
=3 / 26=0.11538 \mathrm{~A}\end{array}$ & & & \\
\hline
\end{tabular}

The circuit is presented on Figure 2 and the components characteristics are listed in Table I, together with the nominal value of mesh currents $\mathrm{W}$ and $\mathrm{Z}$. We will restrict our computations to current $\mathrm{W}$ only, since the procedure is entirely similar for current $\mathrm{Z}$.

We first use the standard BIPM derivative method. The partial derivatives are:

$$
\begin{gathered}
\partial \mathrm{W} / \partial \mathrm{A}=\mathrm{E} .(\partial / \partial \mathrm{x})[(\mathrm{y}+\mathrm{z}) /(\mathrm{x} \cdot \mathrm{y}+\mathrm{y} \cdot \mathrm{z}+\mathrm{z} \cdot \mathrm{x})]=-\mathrm{E} \cdot[(\mathrm{y}+\mathrm{z}) /(\mathrm{x} \cdot \mathrm{y}+\mathrm{y} \cdot \mathrm{z}+\mathrm{z} \cdot \mathrm{x})]^{2}=-(7 / 26)^{2} \\
\partial \mathrm{W} / \partial \mathrm{B}=\mathrm{E} \cdot(\partial / \partial \mathrm{y})[(\mathrm{y}+\mathrm{z}) /(\mathrm{x} \cdot \mathrm{y}+\mathrm{y} \cdot \mathrm{z}+\mathrm{z} \cdot \mathrm{x})]=-\mathrm{E} \cdot[\mathrm{z} /(\mathrm{x} \cdot \mathrm{y}+\mathrm{y} \cdot \mathrm{z}+\mathrm{z} \cdot \mathrm{x})]^{2}=-(4 / 26)^{2} \\
\partial \mathrm{W} / \partial \mathrm{C}=\mathrm{E} \cdot(\partial / \partial \mathrm{z})[(\mathrm{y}+\mathrm{z}) /(\mathrm{x} \cdot \mathrm{y}+\mathrm{y} \cdot \mathrm{z}+\mathrm{z} \cdot \mathrm{x})]=-\mathrm{E} \cdot[\mathrm{y} /(\mathrm{x} \cdot \mathrm{y}+\mathrm{y} \cdot \mathrm{z}+\mathrm{z} \cdot \mathrm{x})]^{2}=-(3 / 26)^{2}
\end{gathered}
$$

The standard formula gives

$$
\begin{gathered}
\sigma_{\mathrm{W}}^{2}=[\partial \mathrm{W} / \partial \mathrm{A}]^{2} \cdot \sigma_{\mathrm{A}}^{2}+[\partial \mathrm{W} / \partial \mathrm{B}]^{2} \cdot \sigma_{\mathrm{B}}^{2}+[\partial \mathrm{W} / \partial \mathrm{C}]^{2} \cdot \sigma_{\mathrm{C}}^{2}= \\
=(7 / 26)^{4} \cdot(0.01333)+(4 / 26)^{4} \cdot(0.03)+(3 / 26)^{4} \cdot(0.05333)=0.000096259 \mathrm{~A}^{2} .
\end{gathered}
$$

The standard deviation is $\sigma_{\mathrm{W}}=\sqrt{ } 0.000096259=0.00981 \mathrm{~A}$.

Then, the BIPM method gives the value $\mathrm{W}=0.27 \pm 0.01 \mathrm{~A}$ to mesh current $\mathrm{W}$.

Now, let us go to the integral method. The joint probability density function is

$$
\mathrm{f}_{\mathrm{ABC}}(\mathrm{x}, \mathrm{y}, \mathrm{z})=\mathrm{f}_{\mathrm{A}}(\mathrm{x}) \cdot \mathrm{f}_{\mathrm{B}}(\mathrm{y}) \cdot \mathrm{f}_{\mathrm{C}}(\mathrm{z})=1 /[(0.4) \cdot(0.6) \cdot(0.8)] \cong 5.2 \Omega^{-3}
$$


The mean $\mathrm{m}_{\mathrm{W}}$ of current $\mathrm{W}$ is calculated by the integral

$$
\mathrm{m}_{\mathrm{W}}=(5.2) \int_{3.6}^{4.4} \int_{2.7}^{3.3} \int_{1.8}^{2.2}\left[\frac{y+z}{x \cdot y+y . z+z \cdot x}\right] \cdot \mathrm{dx} \cdot \mathrm{dy} \cdot \mathrm{dz}=0.269362 \mathrm{~A} .
$$

This value of $m_{w}$ comes out to be very close to, but not identical with, the nominal value. The variance $\sigma_{\mathrm{W}}{ }^{2}$ is calculated next:

$$
\sigma_{\mathrm{W}}^{2}=(5.2) \int_{3.6}^{4.4} \int_{2.7}^{3.3} \int_{1.8}^{2.2}\left[\frac{y+z}{x . y+y . z+z . x}-0.269362\right]^{2} \cdot \mathrm{dx} \cdot \mathrm{dy} \cdot \mathrm{dz}=0.0000974275 \mathrm{~A}^{2}
$$

The standard deviation is $\sigma_{\mathrm{W}}=\sqrt{ } 0.0000974275=0.009870$.

Then, the value of current $\mathrm{W}=0.27 \pm 0.01 \mathrm{~A}$, the same value that the formula of BIPM had given before.

We see that the integral method computes mean and variance as easily as the BIPM procedures. In fact, present technological advances let computer programs like Python or Wolfram Alpha do the boring calculations. The assumed independence of variables also helps in multiple integrations

In preparation for the study of systems with non-differentiable functions, the next section will be about step functions and sigmoids.

\section{Sigmoids}

Sigmoids constitute a family of continuous, differentiable functions that may help to attenuate sudden (and not differentiable) changes in the behavior of functions.

We will start with the step function. In a way, the step function is the black sheep of the sigmoid family, but also a model that all the other members try to imitate. It is also disputable whether the step function may truly belong to the sigmoid family, because it is not itself differentiable. Its definition is

$$
\begin{aligned}
& u(x)=0 \text { for } x<0, \\
& u(x)=1 \text { for } x>0 .
\end{aligned}
$$

We leave the value $u(0)$ unspecified, we do not need to specify it in our study.

The step function was invented by Oliver Heaviside, a British engineer who, from the second half of the nineteenth century to the beginning of the twentieth century, was studying transients in electrical circuits and had therefore to deal with linear differential equations. He found situations in which the available mathematical apparatus to solve his differential equations was not able to cope with situations like: a key suddenly includes a new component or voltage source into the circuit. 
Heaviside acted intuitively and devised the step function as a suitable device, a gadget to solve his problem. Today the step function is also called "the Heaviside step function".

This non-differentiable function was even provided with a kind of derivative, the delta function. The delta function (sometimes associated to the name of Dirac, who used it in his works in quantum theory) is appropriately written as $\delta(\mathrm{x})$, and is a thing that equals zero everywhere except at $x=0$, where it becomes a spike going to infinity. We will not use the delta function here. Of course, and in a broad sense, delta is the derivative of step, and step is the integral of delta.

Mathematicians were becoming increasingly nervous about engineers and physicists using strange devices that they dared call "functions". But the devices inarguably worked! At last, in 1950 Laurent Schwartz published his study on distributions. Distributions allowed rigorous mathematical description of delta and, consequently, of step functions. Thanks to L. Schwartz, deltas became respectable.

Now sporting the proper dress of distribution, the step function could be admitted to the sigmoid family. Let us use it now.

One possible application of step functions is to define the interval on which a given function is alive. Out of the interval, the function is dead, it equals zero. Such is the case of the uniform probability density of a random variable $\mathrm{f}(\mathrm{x})$, which has a constant nonzero value $1 /(b-a)$ over the interval $(a, b)$ and equals zero out of the interval.

Suppose we wish to find the mean value of a certain function $\mathrm{g}(\mathrm{x})$ whose probability density is the function $f(x)=[u(x-a)-u(x-b)] /(b-a)$ just described. We duly write

$$
\text { mean of } \mathrm{g}(\mathrm{x})=\left(\frac{1}{b-a}\right) \int_{-\infty}^{+\infty} g(x) \cdot[u(x-a)-u(x-b)] \cdot d x=\left(\frac{1}{b-a}\right) \int_{a}^{b} g(x) \cdot d x \text {. }
$$

In this case, the effect of the step function merely is to redefine the integration limits from $(-\infty,+\infty)$ to $(a, b)$. We always can (and do) directly write the last expression without much thinking about its origin.

But we must take care. Suppose that function $\mathrm{g}(\mathrm{x})$ itself is defined to hold only for $\mathrm{x}>\mathrm{c}$, where $\mathrm{a}<\mathrm{c}<\mathrm{b}$. Then we face the problem of the product of step functions, because as we write

$$
\text { mean of } \mathrm{g}(\mathrm{x})=\left(\frac{1}{b-a}\right) \int_{-\infty}^{+\infty} g(x) \cdot u(x-c) \cdot[u(x-a)-u(x-b)] . d x
$$

we must ascertain what the products $[\mathrm{u}(\mathrm{x}-\mathrm{c}) \cdot \mathrm{u}(\mathrm{x}-\mathrm{a})]$ and $[\mathrm{u}(\mathrm{x}-\mathrm{c}) \cdot \mathrm{u}(\mathrm{x}-\mathrm{b})]$ actually mean. According to the stated inequalities $\mathrm{a}<\mathrm{c}<\mathrm{b}$, it is easy to see that:

$$
\begin{aligned}
& u(x-c) \cdot u(x-a)=u(x-c) \\
& u(x-c) \cdot u(x-b)=u(x-b)
\end{aligned}
$$

Therefore

$$
\text { mean of } \mathrm{g}(\mathrm{x})=\left(\frac{1}{b-a}\right) \int_{c}^{b} g(x) \cdot d x
$$


As we see, the lower integration limit has been changed from $(x=a)$ to $(x=c)$. A special case of product is the square (and we may find it when computing a variance). Here, we accept that $[u(x)]^{2}=u(x)$. But this is good only for integration, for differentiation the case is more complicated and not of our interest here.

After having presented the unit step function, we now take a look at the members proper of the sigmoid family, generally called $\mathrm{s}(\mathrm{x} ; \mathrm{q})$ and used to simulate or replace nondifferentiable step functions $\mathrm{u}(\mathrm{x})$ with differentiable members of the family. There is available a dozen functions that have been proposed as suitable simulation for a step function.

As seen from the expression $\mathrm{s}(\mathrm{x} ; \mathrm{q})$, a sigmoid carries a parameter $\mathrm{q}>0$ in its definition. Parameter $q$ lets one choose how steep is the transition from zero to one at $x=0$, .The greater the value of q, the closer become the two functions. Intuitively,

$$
\lim _{q \rightarrow \infty}[s(x ; q)]=u(x)
$$

Unfortunately, sigmoids do not help in using the BIPM method for calculating uncertainty propagation. This happens because the method does not operate on the sigmoid itself but on its derivative, and sigmoid's derivatives always show a "bump" at $\mathrm{x}=0$. Worse, the bump's height depends on the arbitrary parameter q. Conclusion: the contribution of the variable that uses a sigmoid to compensate for its undifferentiability becomes completely arbitrary! Not good!

In spite of being of no help in BIPM method, sigmoids may be a viable alternative in integration methods. Let us see it with a specific example: compute the mean of $g(x)=x$ when the probability density is uniform over the interval $a=1$ to $b=3$. The mean is obviously equal to 2 . The expression of the mean is:

$$
\text { mean of } \mathrm{g}(\mathrm{x})=\left(\frac{1}{3-1}\right) \int_{-\infty}^{+\infty}(x) \cdot[u(x-1)-u(x-3)] . d x
$$

Let sigmoids take the place of step functions. We shall use a popular sigmoid, the logistic function that, when used to simulate the step function $u(x-a)$, is written as

$$
\mathrm{s}(\mathrm{x}-\mathrm{a} ; \mathrm{q})=1 /[1+\exp (-\mathrm{q} \cdot(\mathrm{x}-\mathrm{a}))]=[1+\exp (-\mathrm{q} \cdot(\mathrm{x}-\mathrm{a}))]^{-1} .
$$

The result depends on the value of parameter $q$ that has been chosen, therefore it is better to write "mean of $\mathrm{g}(\mathrm{x})(\mathrm{q})$ " to stress such dependency:

$$
\begin{gathered}
\text { mean of } \mathrm{g}(\mathrm{x})(\mathrm{q})=\left(\frac{1}{2}\right) \int_{-\infty}^{+\infty}(x) \cdot[s(x-1 ; q)-s(x-3 ; q)] \cdot d x= \\
=\left(\frac{1}{2}\right) \int_{-\infty}^{+\infty}(x) \cdot\left\{[1+\exp (-q(x-1))]^{-1}-[1+\exp (-q(x-3))]^{-1}\right\} \cdot d x .
\end{gathered}
$$

In this simple example the correct answer is already obtained with $\mathrm{q}=1$ (integration with help of Wolfram Alpha): mean of $g(x)(1)=2$. 
If we change the integrand to a function which itself contains a step function, we already know that this move may simply amount to changing integration limits, because the product of step functions may be easily interpreted as a new step function. Unfortunately, this is not the case with sigmoids: there is no simple rule giving the result of the product of two sigmoids as a new sigmoid of the same kind.

To see an example, let us change the integrand a little bit: $\mathrm{g}(\mathrm{x})=\mathrm{x}$ is replaced by $h(x)=x \cdot u(x-2)$. We know that this move will change the lower integration limit to $x=2$ :

$$
\text { mean of } h(x)=\int_{-\infty}^{+\infty} \frac{x}{2} \cdot \mathrm{u}(\mathrm{x}-2) \cdot[\mathrm{u}(\mathrm{x}-1)-\mathrm{u}(\mathrm{x}-3)] \cdot \mathrm{dx}=\int_{2}^{3} \frac{x}{2} \cdot \mathrm{dx}=1.25
$$

Let now replace all step functions with their corresponding sigmoids (again, logistic functions, all of them with the same value of $q$, for simplicity):

$$
\begin{gathered}
\text { mean of } \mathrm{h}(\mathrm{x})(\mathrm{q})= \\
=(0.5) \int_{-\infty}^{+\infty} x \cdot[1+\exp (-q(x-2))]^{-1} \cdot\left\{[1+\exp (-q(x-1))]^{-1}-[1+\right. \\
\left.\exp (-\mathrm{q}(\mathrm{x}-3))]^{-1}\right\} \cdot d x
\end{gathered}
$$

Table II - Results for mean of $h(x)=x . u(x-2)$

\begin{tabular}{|c|c|}
\hline $\begin{array}{c}\text { value of } \\
\text { parameter } \mathbf{q}\end{array}$ & $\begin{array}{c}\text { calculated } \\
\text { value of } \\
\text { the mean of } \\
\mathbf{h}(\mathbf{x})\end{array}$ \\
\hline 1 & 1.54099 \\
\hline 2 & 1.32826 \\
\hline 4 & 1.25933 \\
\hline 8 & 1.25017 \\
\hline 16 & 1.25 \\
\hline
\end{tabular}

Results after computation as shown in Table II as a function of q. It is seen how the integral converges to the exact value as $q$ increases.

Another experiment may be performed by fixing the integration limits from $\mathrm{x}=1$ (careful, here!) to $\mathrm{x}=3$, and only keeping one sigmoid, the one native to $\mathrm{h}(\mathrm{x})$ :

$$
\text { mean of } \mathrm{h}(\mathrm{x})(\mathrm{q})=(0.5) \cdot \int_{1}^{3} x \cdot[1+\exp (-q(x-2))]^{-1} \cdot \mathrm{dx}
$$


This new experiment appears to be a kind of hybrid (and simplified) application of sigmoids; we obviously expect results similar to those from the preceding experiment. The numerical results, function of q, are shown in Table III.

Table III- Results for mean of $h(x)=x . u(x-2)$ computed with a hybrid/simplified application

\begin{tabular}{|c|c|}
\hline $\begin{array}{c}\text { value of } \\
\text { parameter q }\end{array}$ & $\begin{array}{c}\text { calculated } \\
\text { value of } \\
\text { the mean of } \\
\mathbf{h}(\mathbf{x})\end{array}$ \\
\hline 1 & 1.07944 \\
\hline 2 & 1.1406 \\
\hline 4 & 1.20427 \\
\hline 8 & 1.2372 \\
\hline 16 & 1.24679 \\
\hline 32 & 1.2492 \\
\hline 64 & 1.2498 \\
\hline 128 & 1.24995 \\
\hline 256 & 1.24999 \\
\hline 512 & 1.25 \\
\hline
\end{tabular}

Convergence is slower here but in practice the result for $\mathrm{q}=16$ could already be rounded to 1.25. The computations were repeated until Wolfram Alpha finally decided to show 1.25 !

In summary, sigmoids may be used in integral methods, if so desired, as replacements for step functions. In practical use it is not necessary to follow the process of convergence, as shown above as an illustration. Just give to parameter q some value of a few hundreds or thousands!

\section{A water-heating system}

As it was mentioned before, the standard procedure for studying uncertainty propagation is dependent on the fact that the measurand be differentiable with respect to its variables. If not, no uncertainty propagation study can be performed. But who knows whether our integration method could help in this case? We will see it now, and we shall conduct our study around a specific example.

Either in the domestic or in the industrial environment we find devices like heaters, air conditioners and similar appliances that are provided with a thermostat that connects or 
disconnects the device in order to keep some temperature within narrow limits. And a thermostat (an on-off switch) introduces a non-differentiable function into the picture! The example we are about to study is an electric water heater whose resistor is disconnected every time the water supply goes below certain established value.

First of all, please meet our friend Jimmy. Jimmy lives in an old apartment building whose plumbing was very badly designed, so badly that, when someone opens or closes a faucet, the water flow in the neighboring apartments is affected by this action. And vice-versa, of course. Conclusion: Jimmy's apartment water distribution is sensitive to how and how much all the other tenants use water.

Also, there is no central heating nor gas in the building. In order to get a hot shower, Jimmy had an electric water heater installed in the pipe that feeds his bathroom. But when people next door flushed their toilet, water flow to Jimmy's shower temporarily halted and the heater's resistor, no more immersed in running water, burnt. To prevent this disaster, a flow detector was installed in the pipe, coupled with an electrical on-off switch that disconnected the electrical supply to the resistor whenever the flow fell below a certain safe value (safe with regard to the resistor's health). Figure 3 shows a simplified schematic diagram of the contraption just described.

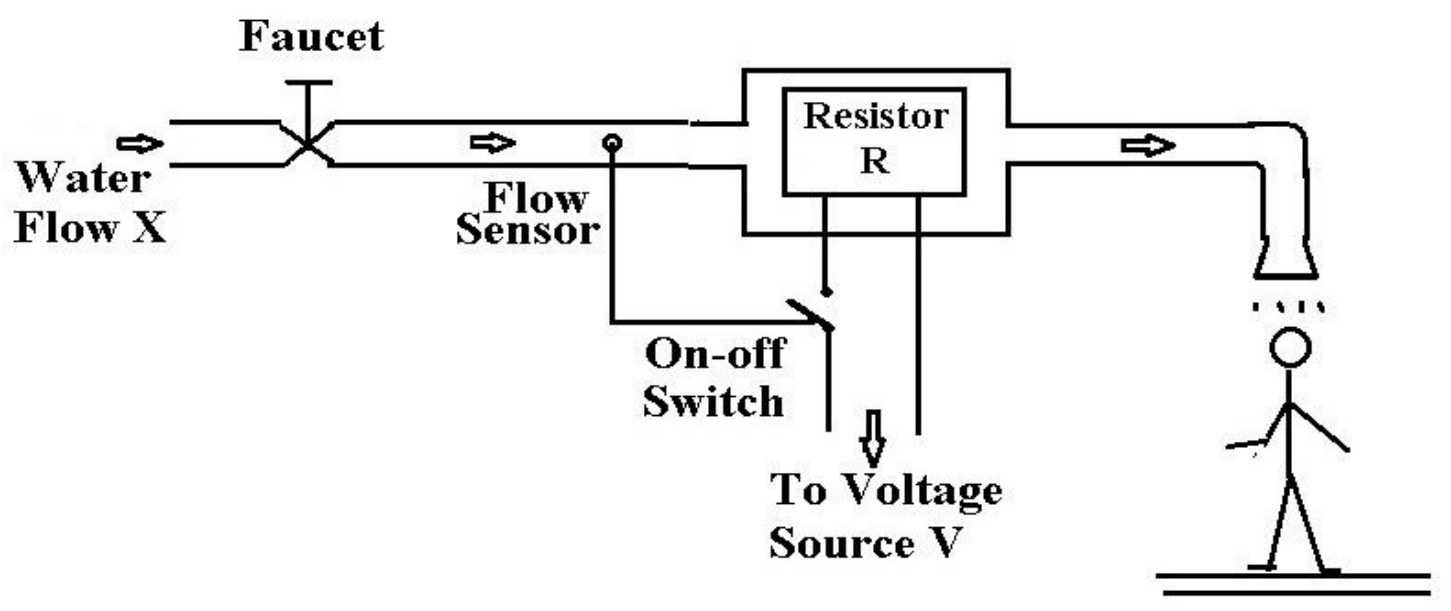

Fig.3 - A water-heating system that includes a switch to cut off the electrical power to the heating resistor when the flow of water goes below a critical value.

Based on the described scenario, we desire to provide Jimmy with a consolation prize: the mean value and the standard deviation of the temperature rise in his shower's water. To this purpose, we shall perform an uncertainty propagation study of the system depicted on Figure 3 to demonstrate how the integral approach may deal with a case of nondifferentiable function.

We treat the water flow as a random variable (X). For completeness, we also consider the voltage supply (V) as a second random variable. The heating resistor $\mathrm{R}$ is fixed, not a r,v. 
The measurand is the water temperature rise (Y), a r.v. whose mean value and variance are to be computed from $\mathrm{X}$ and $\mathrm{V}$.

A few simplifications are made to the problem: it is supposed that the whole of the heat generated in resistor $\mathrm{R}$ goes to raise water temperature. Water input temperature $t_{\text {in }}$ is raised to the final value $t_{\text {out }}=t_{\text {in }}+Y$. It is also supposed that heating and cooling of water are instantaneous processes, that there is no thermal inertia to slow down the processes. Therefore, if a neighbor flushing the toilet causes resistor $\mathrm{R}$ to be cut off from the electrical supply, then instantaneously $t_{\text {out }}$ becomes equal to $t_{\text {in. }}$. When the flow comes back to a value above the critical value, then the corresponding $t_{\text {out }}$ is instantaneously obtained. The model is a crude representation of the real case but, of course, we wish to discuss uncertainty propagation, not heat propagation!

In a steady-state situation, when resistor $\mathrm{R}$ in connected to the electrical network, the following conservation equation holds:

$$
\begin{gathered}
\text { electrical power input }= \\
=(\text { fluid mass flow })(\text { fluid specific heat })(\text { temperature difference }),
\end{gathered}
$$

therefore

$$
\begin{gathered}
\text { temperature difference }= \\
=(\text { electrical power input }) /(\text { fluid mass flow })(\text { fluid specific heat }) .
\end{gathered}
$$

When the fluid is water, and the system of units is the SI, the equation becomes

$$
\begin{gathered}
\text { temperature difference in kelvins }= \\
=(\text { input power in watts })(4.18 \times \text { water flow in liters per second })
\end{gathered}
$$

or, in more practical units:

temperature difference in degree celsius $=$ $=$ (input power in watts)(251 x water flow in liters per minute).

Then we may write that in a normal situation:

$$
\mathrm{Y}=\mathrm{V}^{2} /(251 . \mathrm{R} . \mathrm{X})
$$

with the following units: $\mathrm{Y}-->{ }^{\circ} \mathrm{C} ; \mathrm{V}-->\mathrm{V} ; \mathrm{R}-->\Omega ; \mathrm{X}-->\mathrm{L} / \mathrm{min}$.

At this point, certain questions arise whose answers are vital to our understanding of the problem. Since the connection of resistor $\mathrm{R}$ to the voltage source $\mathrm{V}$ is controlled by the flow of water $X$, does not this fact imply that $V$ becomes a function of $X$ ? So that we no more may consider $\mathrm{V}$ and $\mathrm{X}$ as independent variables? And we can no more accept their joint probability density $f_{X V}(x, v)$ being given by $f_{X}(x) \cdot f_{V}(v)$ ?

However, a closer examination shows that the behavior of $\mathrm{X}$ does not influence $\mathrm{V}$, directly or indirectly, but instead it has effect directly on Y. In other words, that we must write the complete definition of $\mathrm{Y}$ as

$$
\begin{gathered}
\mathrm{Y}=\left[\mathrm{V}^{2} /(251 . R \cdot \mathrm{X})\right] \text { when } \mathrm{x} \geq \mathrm{x}_{\mathrm{c}} \\
\mathrm{Y}=0 \text { when } \mathrm{x}<\mathrm{x}_{\mathrm{c}} .
\end{gathered}
$$


Or:

$$
\mathrm{Y}=\mathrm{V}^{2} \cdot \mathrm{u}\left(\mathrm{x}-\mathrm{x}_{\mathrm{c}}\right) /(251 \cdot \mathrm{R} \cdot \mathrm{X})
$$

In fact, when the flow is below the critical value, $\mathrm{V}$ stays as usual, but $\mathrm{Y}$ goes to zero. This means that if the lower limit of integration on $\mathrm{x}$ is less than $\mathrm{x}_{\mathrm{c}}$, the integration on $\mathrm{x}$ gives zero as result. This certainly happens when the user of the water-heater closes the faucet such that the resulting flow diminishes enough or completely ceases.

Integration with respect to $\mathrm{V}$ is performed normally. Because of independence, the integration with respect to $\mathrm{V}$ becomes simply a factor in the final expression.

Let the following symbols and concepts be defined:

1) $x, v=$ dummy variables for integration;

2) $\mathrm{X}_{\mathrm{m}}=$ minimum value of water flow; it could be, but not necessarily is, equal to 0 ;

3) $\mathrm{x}_{\mathrm{c}}=$ critical value of flow, at which the transition to and from 1 occurs in $\mathrm{u}\left(\mathrm{x}-\mathrm{x}_{\mathrm{c}}\right)$;

4) $\mathrm{x}_{\mathrm{M}}=$ greatest value of flow, established by opening more or less the faucet;

5) $\mathrm{x}_{\mathrm{M}}-\mathrm{x}_{\mathrm{m}}=$ interval over which the probability density function of flow is uniform;

6) It is expected that in a normal situation the following inequalities always hold:

$$
0 \leq \mathrm{x}_{\mathrm{m}}<\mathrm{x}_{\mathrm{c}}<\mathrm{x}_{\mathrm{M}}
$$

7) $f_{X}(x)=\left[u\left(x-x_{m}\right)-u\left(x-x_{M}\right)\right] /\left(x_{M}-x_{m}\right)=$ the probability density function of $X$; the action of the step function in $\mathrm{Y}$ will reduce the limits of integration to $\left(\mathrm{x}_{\mathrm{M}}, \mathrm{x}_{\mathrm{c}}\right)$;

8) Supply voltage V has nothing special about itself and is independent from X. Its probability density $f_{V}(v)$ is uniform over the interval $\left(v_{M}-v_{m}\right)$, then

and $f_{v}(v)=0$ out of the interval.

$$
f_{V}(v)=1 /\left(v_{M}-v_{m}\right) \text { over }\left(v_{m}, v_{M}\right) \text {. }
$$

9) Since variables $X$ and $V$ are independent from each other, their joint probability density $f_{X V}(x, v)=f_{X}(x) \cdot f_{V}(v)$.

We will compute mean and variance of temperature rise $\mathrm{Y}$ for a specific numerical case. The following numerical values have been chosen for this example:

$\mathrm{x}_{\mathrm{M}}=5.0 \mathrm{~L} / \mathrm{min}$

$\mathrm{X}_{\mathrm{m}}=0.5 \mathrm{~L} / \mathrm{min}$;

$\mathrm{x}_{\mathrm{c}}=1.0 \mathrm{~L} / \mathrm{min}$

voltage is $220 \mathrm{~V} \pm 10 \%$, giving $\mathrm{v}_{\mathrm{m}}=198 \mathrm{~V}$ and $\mathrm{v}_{\mathrm{M}}=242 \mathrm{~V}$, uniformly distributed; $\mathrm{R}=6.4 \Omega$, fixed.

Nominal heater power $=(220)^{2} / 6.4=7.56 \mathrm{~kW}$

Nominal temperature rise at critical flow $\mathrm{x}_{\mathrm{c}}=1.0 \mathrm{~L} / \mathrm{min}-->7560 / 251 / 1 \cong 30{ }^{\circ} \mathrm{C}$

Nominal temperature rise at maximum flow $\mathrm{x}_{\mathrm{M}}=5.0 \mathrm{~L} / \mathrm{min}-->7560 / 251 / 5=6{ }^{\circ} \mathrm{C}$ 
Then:

$\mathrm{f}_{\mathrm{X}}(\mathrm{x})=1 /\left(\mathrm{x}_{\mathrm{M}}-\mathrm{x}_{\mathrm{m}}\right)=1 /(5-0.5)=1 / 4.5 \mathrm{~min} / \mathrm{liter}$;

$\mathrm{f}_{\mathrm{V}}(\mathrm{v})=1 /\left(\mathrm{v}_{\mathrm{M}}-\mathrm{v}_{\mathrm{m}}\right)=1 /(242-198)=1 / 44 \mathrm{~V}^{-1}$

$f_{x v}(x, v)=f_{X}(x) \cdot f_{v}(v)=0.00505051 \mathrm{~min} \cdot L^{-1} \cdot V^{-1}$

Other constant factors in integration:

$$
\begin{gathered}
\text { 1/[251.R. } \left.\left(v_{M}-v_{m}\right) .\left(x_{M}-x_{m}\right)\right]=3.14 \times 10^{-6} \\
f_{x v}(x, v) /(251 . R)^{2}=1.9571668 \times 10^{-9}
\end{gathered}
$$

The mean value $\mathrm{m}_{\mathrm{Y}}$ of temperature rise $\mathrm{Y}$ is given by:

$$
\begin{gathered}
\mathrm{m}_{\mathrm{Y}}=\int_{v m}^{v M} \cdot \int_{x m}^{x M}\left[\mathrm{v}^{2} /(251 \cdot \mathrm{R} \cdot \mathrm{x})\right] \cdot \mathrm{u}\left(\mathrm{x}-\mathrm{x}_{\mathrm{c}}\right) \cdot \mathrm{f}_{\mathrm{Xv}}(\mathrm{x}, \mathrm{v}) \cdot \mathrm{dx} \cdot \mathrm{dv}= \\
=\left\{1 /\left[251 \cdot \mathrm{R} \cdot\left(\mathrm{v}_{M}-\mathrm{v}_{\mathrm{m}}\right) \cdot\left(\mathrm{x}_{M}-\mathrm{x}_{\mathrm{m}}\right)\right]\right\} \cdot \int_{v m}^{v M} \cdot \int_{x c}^{x M}\left(\mathrm{v}^{2} / \mathrm{x}\right) \cdot \mathrm{dx} \cdot \mathrm{dv}= \\
=3.14 \times 10^{-6} \cdot \int_{198}^{242} \cdot \int_{1}^{5}\left(\mathrm{v}^{2} / \mathrm{x}\right) \cdot \mathrm{dx} \cdot \mathrm{dv}=10.7981^{\circ} \mathrm{C} .
\end{gathered}
$$

Then, the constant

$$
(2) \cdot \mathrm{m}_{\mathrm{Y}} \cdot \mathrm{f}_{\mathrm{Xv}}(\mathrm{x}, \mathrm{v}) /(251 . \mathrm{R})=6.78983 \times 10^{-5} \text {. }
$$

In the computation of variance $\sigma_{Y}^{2}$ there is a squared unit step function. We already know that, for integration purposes, a squared unit step function behaves as if it were not squared. Its only effect appears in changing the lower limit of integration from $\mathrm{x}=\mathrm{x}_{\mathrm{m}}$ to $\mathrm{x}=\mathrm{x}_{\mathrm{c}}$.

$$
\begin{gathered}
\sigma \mathrm{Y}^{2}=\int_{v m}^{v M} \cdot \int_{x m}^{x M}\left[\mathrm{Y}-\mathrm{m}_{\mathrm{Y}}\right]^{2} \cdot \mathrm{f}_{\mathrm{Xv}}(\mathrm{x}, \mathrm{v}) \cdot \mathrm{dx} \cdot \mathrm{dv}= \\
=\mathrm{f}_{\mathrm{xv}}(\mathrm{x}, \mathrm{v}) \cdot \int_{v m}^{v M} \cdot \int_{x m}^{x M}\left\{\left[\left(\mathrm{v}^{2} \cdot \mathrm{u}\left(\mathrm{x}-\mathrm{x}_{\mathrm{c}}\right)\right) /(251 \cdot \mathrm{R} \cdot \mathrm{x})\right]-\mathrm{m}_{\mathrm{Y}}\right\}^{2} \cdot \mathrm{dx} \cdot \mathrm{dv} .
\end{gathered}
$$

After expanding the square, the variance is given by the algebraic sum of three quantities (I1, I2, I3) which will be computed separately:

$\sigma_{\mathrm{Y}}^{2}=\mathrm{I} 1+\mathrm{I} 2+\mathrm{I} 3$

$\mathrm{I} 1=\mathrm{f}_{\mathrm{xv}}(\mathrm{x}, \mathrm{v}) \cdot \int_{v m}^{v M} \cdot \int_{x m}^{x M}\left\{\left[\left(\mathrm{v}^{2} \cdot \mathrm{u}\left(\mathrm{x}-\mathrm{x}_{\mathrm{c}}\right)\right) /(251 \cdot \mathrm{R} \cdot \mathrm{x})\right]\right\}^{2} \cdot \mathrm{dx} \cdot \mathrm{dv}$

$\mathrm{I} 2=-\mathrm{f}_{\mathrm{xv}}(\mathrm{x}, \mathrm{v}) \cdot \int_{v m}^{v M} \cdot \int_{x m}^{x M}\left\{2 \cdot m_{y} \cdot\left[\left(\mathrm{v}^{2} \cdot \mathrm{u}\left(\mathrm{x}-\mathrm{x}_{\mathrm{c}}\right)\right) /(251 \cdot \mathrm{R} \cdot \mathrm{x})\right]\right\} \cdot \mathrm{dx} \cdot \mathrm{dy} ;$

$\mathrm{I} 3=\mathrm{f}_{\mathrm{xv}}(\mathrm{x}, \mathrm{v}) \cdot \int_{v m}^{v M} \cdot \int_{x m}^{x M} m_{y}^{2} \cdot d x \cdot d v$.

Then:

$$
\mathrm{I} 1=\left(1.9571668 \times 10^{-9} \int_{v m}^{v M} \int_{x c}^{x M} \frac{v^{4}}{x^{2}} \cdot d x \cdot d v=164.615\right.
$$




$$
\begin{gathered}
\mathrm{I} 2=-\left(6.78983 \times 10^{-5}\right) \cdot \int_{v m}^{v M} \int_{x c}^{x M} \frac{v^{2}}{x} \cdot d x, d v=-233.494 \\
\mathrm{I} 3=\mathrm{m}_{\mathrm{y}}^{2}=(10.7981)^{2}=116.598963
\end{gathered}
$$

Finally: $\sigma_{\mathrm{Y}}^{2}=47.72$ and $\sigma_{\mathrm{Y}}=6.91$

At last, our friend Jimmy may be informed about the statistical characteristics of his water-heating system:

$$
\mathrm{t}_{\text {out }}=\mathrm{t}_{\text {in }}+10.8 \pm 6.9\left({ }^{\circ} \mathrm{C}\right)
$$

$* * * * * * * * * * * * * * * * * * * * * * * * * * * * * * * * * * * * * *$

So, we finished an example on the use of step functions to treat certain non-differentiable functions. Would sigmoids be of any help in dealing with this or similar jobs? To complete our work, we now investigate what would happen when step functions are replaced by sigmoids in the problem of water heater.

We already have seen that the results depend on the value chosen for parameter $q$ and that by increasing $\mathrm{q}$ one gets results nearer to those given by the direct use of step functions.

We again use the logistic function as a suitable sigmoid and the hybrid/simplified expression in computations.

The mean of $Y(q)$ is given by:

$$
\text { mean of } \mathrm{Y}(\mathrm{q})=\int_{v m}^{v M} \cdot \int_{x m}^{x M}\left[\mathrm{v}^{2} /(251 \cdot \mathrm{R} \cdot \mathrm{x})\right] \cdot[1+\exp (-\mathrm{q}(\mathrm{x}-1))]^{-1} \cdot \mathrm{f}_{\mathrm{XV}}(\mathrm{x}, \mathrm{v}) \cdot \mathrm{dx} \cdot \mathrm{dv} \text {. }
$$

The integral quickly converges: mean of $\mathrm{Y}(512)=10.7981$

In the calculation of the variance, sigmoids may be very helpful, because the calculation with step function is rather complex and long. With sigmoid, the burden of computation stays mostly with the integration machine, which is presented with the following integrand:

$$
\begin{gathered}
\text { variance of } \mathrm{Y}(\mathrm{q})= \\
=\mathrm{f}_{\mathrm{xv}}(\mathrm{x}, \mathrm{v}) \cdot \int_{v m}^{v M} \cdot \int_{x m}^{x M}\left\{\left[\left(\mathrm{v}^{2}\right) \cdot[1+\exp (-\mathrm{q}(\mathrm{x}-1))]^{-1} /(251 \cdot \mathrm{R} \cdot \mathrm{x})\right]-\mathrm{m}_{\mathrm{Y}}\right\}^{2} \cdot \mathrm{dx} \cdot \mathrm{dv} .
\end{gathered}
$$

Convergence is much slower now, but variance of $\mathrm{Y}(8000)=47.718$

In summary, sigmoids may represent useful devices to simplify some complicated situations involving step functions. 


\section{Implicit functions}

Sometimes the measurand happens to be an implicit function. This case is covered by BIPM (JCGM 103 CD; 2018-10-04; Guide to the expression of uncertainty in measurement - Developing and using measurement models).

Modeling with implicit functions is more complicated than with the more usual explicit functions. In this section, we will see how the integral method can handle an implicit measurand. The subject matter will be discussed with the help of an example.

Let us go back to the circuit of Figure 1. To deal with a more descriptive case we suppose that resistor $r$ in it also is a r.v. (nominal value $r=1 \Omega$; variance $\sigma_{\mathrm{r}}^{2}=0.00333 \Omega^{2}$ ). It is already known the nominal value of the current $\mathrm{I}=0.0909 \mathrm{~A}$. With differential or integrating method we find the variance of current: $\sigma_{\mathrm{I}}^{2}=0.0000230 \mathrm{~A}^{2}$.

This done, suppose a new situation, in which resistor $\mathrm{R}$ increases its resistance when heated by current I. The model has now changed to a different one, in which resistor $\mathrm{R}$ becomes a function of current $I$ and is therefore written as $R(I)$.

In consequence, the former explicit function $I=E /(r+R)$ is transformed into a different function $\mathrm{I}=\mathrm{E} /(\mathrm{r}+\mathrm{R}(\mathrm{I}))$ that clearly is implicit because the current appears on both sides of the equation.

To be more specific, suppose that the resistance of resistor $\mathrm{R}$ changes linearly with the power R.I ${ }^{2}$ generated in it by the current: $R=R_{0}\left(1+\alpha \cdot R \cdot I^{2}\right)=R_{0} /\left(1-\alpha \cdot R_{0} \cdot I^{2}\right)$.

The new symbol $R_{0}$ represents the value of $R$ at room temperature and when no current circulates in it. The role of r.v. is now transferred to $R_{0}$ (as before, nominal value $R_{0}=$ $10 \Omega$, variance $\sigma_{\mathrm{R} 0}{ }^{2}=0.333 \Omega^{2}$ ).

When the expression of the current-dependent $\mathrm{R}$ is inserted in the equation of $\mathrm{I}$, the result is

$$
\mathrm{I}=\mathrm{E} /\left[\mathrm{r}+\left(\mathrm{R}_{0} /\left(1-\alpha \cdot \mathrm{R}_{0} \cdot \mathrm{I}^{2}\right)\right)\right]
$$

After simplification, one gets the final expression of the implicit equation:

$$
\alpha . r . R_{0} \cdot I^{3}-\mathbf{E} \cdot \alpha \cdot \mathbf{R}_{0} \cdot I^{2}-\left(r+R_{0}\right) . I+E=0 .
$$

In conclusion, we got a cubic equation to implicitly define the current.

What has happened? It has happened that, due to the heating and value change of a resistor, the former explicit function $I=f(R)$ has given its place to an implicit function $\mathrm{g}\left[\mathrm{R}_{0}, \mathrm{I}\left(\mathrm{R}_{0}\right)\right]=0$.

To find the current we need the roots of the polynomial, after replacing variables and parameters with their nominal values $\left(\mathrm{E}=1 \mathrm{~V} ; \mathrm{r}=1 \Omega ; \mathrm{R}_{0}=10 \Omega ; \alpha=2 \Omega^{-1} . \mathrm{A}^{-2}\right.$ ). The value of parameter $\alpha$ was chosen so as to gently warm the resistor.

After this substitution, the polynomial numerically becomes

$$
\text { 20.I } I^{3}-20 . I^{2}-11 . I+1=0 .
$$

Solution of this cubic polynomial gives three real roots: $\mathrm{I}=0.080 ; \mathrm{I}=-0.454 ; \mathrm{I}=1.374$. Only the first root is physically possible in the given circuit, so we keep it and discard the other two roots. Then $\mathrm{I}=0.080 \mathrm{~A}$ becomes the nominal value for this case. 
As we have done before in this paper, first we apply the standard BIPM method whose results we keep as a reference, then we go to the integral method. The BIPM method must be duly adapted to the specific case of implicit functions, as follows. First, take the implicit derivatives, then compute them numerically for nominal values:

$$
\begin{aligned}
& \left(\partial / \partial \mathrm{R}_{0}\right)\left(2 \cdot \mathrm{R}_{0} \cdot \mathrm{I}^{3}-2 \cdot \mathrm{R}_{0} \cdot \mathrm{I}^{2}-\mathrm{R}_{0} \cdot \mathrm{I}-\mathrm{I}+1\right)=0, \\
& {\left[6 \cdot \mathrm{R}_{0} \cdot \mathrm{I}^{2} .\left(\partial \mathrm{I} / \partial \mathrm{R}_{0}\right)+2 \cdot \mathrm{I}^{3}\right]-\left[4 \cdot \mathrm{R}_{0} \cdot \mathrm{I} \cdot\left(\partial \mathrm{I} / \partial \mathrm{R}_{0}\right)+2 \cdot \mathrm{I}^{2}\right]-\left[\mathrm{R}_{0} \cdot\left(\partial \mathrm{I} / \partial \mathrm{R}_{0}\right)+\mathrm{I}\right]-\left(\partial \mathrm{I} / \partial \mathrm{R}_{0}\right)=0 \text {, }} \\
& \partial \mathrm{I} / \partial \mathrm{R}_{0}=\left\{\left[2 . \mathrm{I}^{3}-2 . \mathrm{I}^{2}-\mathrm{I}\right] /\left.\left[1-\mathrm{R}_{0}\left(6 . \mathrm{I}^{2}-4 . \mathrm{I}-1\right)\right\}\right|_{\mathrm{R} 0=10 ; \mathrm{I}=0.080}=-0.00663 \text { A. } \Omega^{-1}\right. \text {. } \\
& (\partial / \partial \mathrm{r})\left(20 . r . \mathrm{I}^{3}-20 . \mathrm{I}^{2}-\mathrm{r} . \mathrm{I}-10 . \mathrm{I}+1\right)=0, \\
& {\left[60 . r . \mathrm{I}^{2} .(\partial \mathrm{I} / \partial \mathrm{r})+20 . \mathrm{I}^{3}\right]-40 . \mathrm{I} .(\partial \mathrm{I} / \partial \mathrm{r})-\mathrm{r} .(\partial \mathrm{I} / \partial \mathrm{r})-10 .(\partial \mathrm{I} / \partial \mathrm{r})-\mathrm{I}=0,} \\
& \partial \mathrm{I} / \partial \mathrm{r}=\left.\left\{\left[20 . \mathrm{I}^{3}-\mathrm{I}\right] /\left[\left(1-60 . \mathrm{I}^{2}\right) \cdot \mathrm{r}+40 . \mathrm{I}-10\right]\right\}\right|_{\mathrm{r}=1 ; \mathrm{I}=0.080}=-0.00505 \mathrm{~A} . \Omega^{-1} \text {. }
\end{aligned}
$$

The variance of current results to be

$$
\begin{gathered}
\sigma_{\mathrm{I}}^{2}=(\partial \mathrm{I} / \partial \mathrm{r})^{2} \cdot \sigma_{\mathrm{r}}^{2}+(\partial \mathrm{I} / \partial \mathrm{R})^{2} \cdot \sigma_{\mathrm{R}}^{2}= \\
(-0.00505)^{2} .(0.00333 . .)+(-0.00664)^{2} .(0.333)=0.00001478 \mathrm{~A}^{2}
\end{gathered}
$$

So much for the differential method. And the integral method? Unfortunately, it does not appear to exist any simple, general rule to perform integrations when an implicit function is involved. However, in the following we will try to adapt the integral method to the example just discussed. Let us see what happens.

We start the discussion by trying to compute the mean of current by its definition as the integral $m_{I}=\iint I . f\left(r, R_{0}\right) \cdot d r . d R_{0}$, where $f\left(r, R_{0}\right)$ is the joint probability density of the two r.v. $\left(r, R_{0}\right)$.

Given that the current has no explicit expression, we could naively replace the integrand by its implicit expression as $m_{I}=\iint\left[\alpha \cdot r \cdot R_{0} \cdot I^{3}-E \cdot \alpha \cdot R_{0} \cdot I^{2}-\left(r+R_{0}\right) \cdot I+E\right] . f\left(r, R_{0}\right) \cdot d r \cdot d R_{0}$. Even now there is no indication of functional dependence of I from the r.v., therefore I is considered a constant by any integral calculator. But in reality the current changes (implicitly!) as the variables go thru their respective integration intervals. In conclusion, this naive approach is wrong.

Let us try a different approach.

The expression $m_{I}=\iint I . f\left(r, R_{0}\right)$.dr.dR $R_{0}$ is formally right, no doubt about it; it is the integration machinery that must be adapted to the case.

For simplicity, let resistor $r$ temporarily return to be a constant, no more a r.v., so that we might deal with the easier problem of a single integral $m_{I}=\int \operatorname{I} . f\left(R_{0}\right) \cdot d R_{0}$.We will also compute the variance $\sigma_{\mathrm{I}}^{2}=\int\left[\mathrm{I}-\mathrm{m}_{\mathrm{I}}\right]^{2} \mathrm{f}\left(\mathrm{R}_{0}\right) \cdot \mathrm{dR} \mathrm{R}_{0}$.

If we are successful in our attempt with a simple integral, we will extend the results to the case of multiple integrals.

For an easier conversation with computer programs some change in symbols is advisable: $\mathrm{R}_{0-->} \mathrm{y}, \mathrm{I}-->$ w. Symbol $\mathrm{x}$ is saved for future use to represent $\mathrm{r}$. 
The expression of $\mathrm{w}$ is computed with $\mathrm{E}=1, \mathrm{r}=1, \alpha=2$, giving

$$
g(y)=(2) \cdot y \cdot w^{3}-(2) \cdot y \cdot w^{2}-(1+y) \cdot w+1
$$



Fig.4 - Plot of current during the integration process

Our integrals are:

$$
\begin{gathered}
\text { mean } \mathrm{m}_{\mathrm{I}}=\int \mathrm{w} \cdot \mathrm{f}(\mathrm{y}) \mathrm{dy} \\
\text { variance } \sigma_{\mathrm{I}}^{2}=\int\left[\mathrm{w}-\mathrm{m}_{\mathrm{I}}\right]^{2} . \mathrm{f}(\mathrm{y}) \cdot \mathrm{dy},
\end{gathered}
$$

with $\mathrm{f}(\mathrm{y})=0.5$ over the integration interval $\mathrm{y}=9$ to $\mathrm{y}=11$.

We start with the mean. Since we wish to integrate numerically, we note that a numerical integral calculator always makes the variable y go thru the integration interval in discrete fashion, that is, in steps. For example, the interval is divided in $\mathrm{N}$ parts, giving $\mathrm{N}+1$ steps when y goes from $y=9$ to $y=11$. Then, some formula is applied, like the Simpson rule, to compute the area under the curve.

We take advantage of this discretization, that the computer necessarily does, in the following way. When $y$ is at the k-th step (that is, $y=y_{k}$ ), the roots of $g\left(y_{k}\right)=0$ are found and the useful root $w_{k}$ is stored in an appropriate place together with the corresponding $\mathrm{y}_{\mathrm{k}}$ 


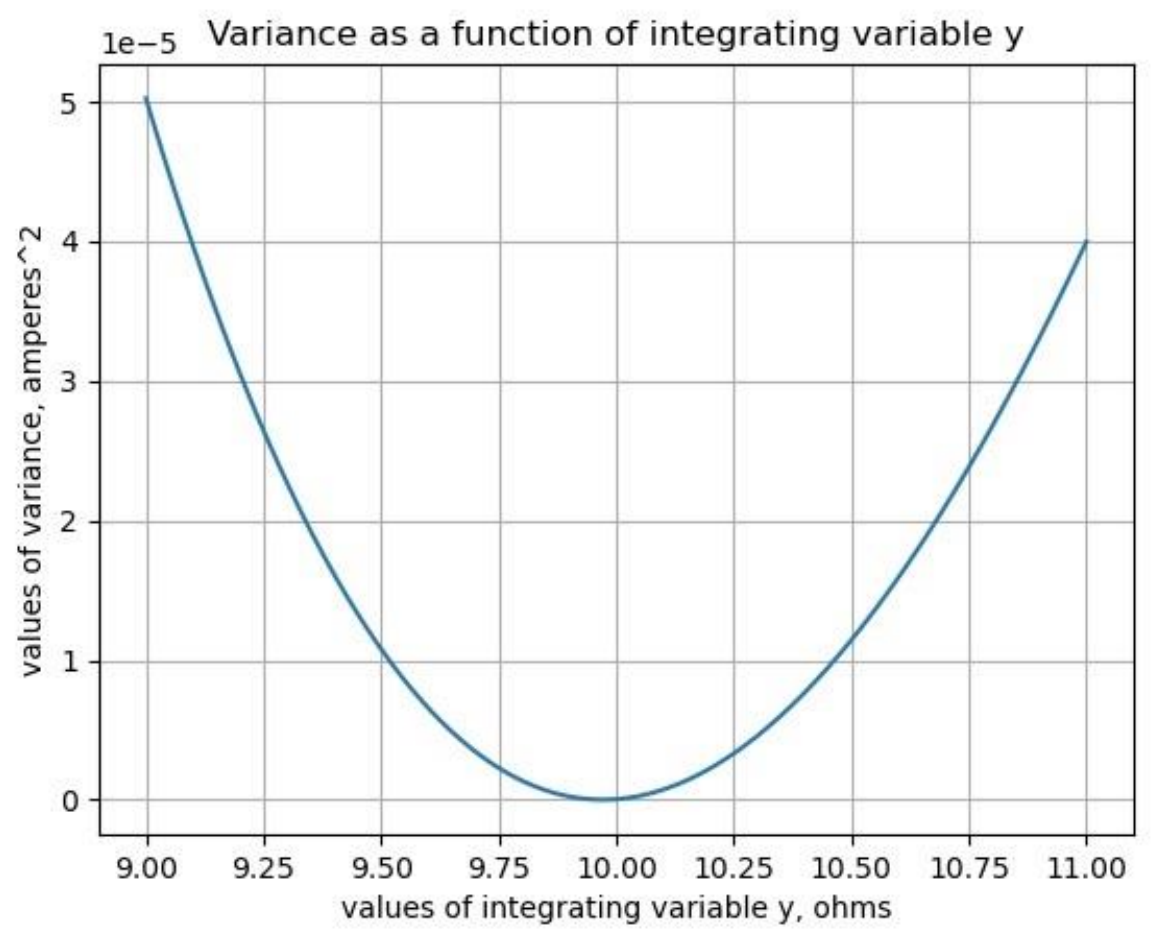

Fig.5 - Plot of variance during the integration process

In other words, a set is created such that it contains all the pairs $\left(\mathrm{y}_{\mathrm{k}}, \mathrm{w}_{\mathrm{k}}\right)$ of running variable and corresponding value of current. This means that the problem of implicit function is solved locally at each integration step.

Of course, a computer is needed: if there are one hundred steps, there also are one hundred root-findings of a cubic to do! This program has been implemented in Python. Python also offers the possibility of plotting some results. Figure 4 shows the values of $w_{k}$ versus $y_{k}$ as continuous line going thru the points. The area under the curve, multiplied by 0.5 (the probability density), is the mean correctly calculated by integration. The result is not very sensitive to the number of samples: with 21 samples, $m_{I}=0.0803526$; with 101 samples, $\mathrm{m}_{\mathrm{I}}=0.0803525$

Once the mean is obtained, the process is repeated to calculate the variance. Figure 5 shows the plot of $\left(\mathrm{w}_{\mathrm{k}}-\mathrm{m}_{\mathrm{I}}\right)^{2}$ versus $\mathrm{y}_{\mathrm{k}}$, again pictured as a continuous process and calculated with 101 samples. Again, the area under the curve, multiplied by 0.5, gives the variance of the current, correctly calculated by integration: $\sigma_{\mathrm{I}}^{2}=0.00001493 \mathrm{~A}^{2}$.

These results compare favorably with the nominal value $\mathrm{I}=0.080 \mathrm{~A}$ and with the variance of current as calculated following the BIPM rules:

$$
(\partial \mathrm{I} / \partial \mathrm{R})^{2} \cdot \sigma_{\mathrm{R}}^{2}=(-0.00664)^{2} \cdot(0.333)=0.0000147 \mathrm{~A}^{2} .
$$


Looking at Figure 4 one gets the impression that the current is a nearly linear function of variable $y$ in the interval of integration. What if we take this impression as a true fact? If we do, we could "deimplicit" the implicit function once for all into a simpler straight line like $I=A \cdot R_{0}+B$, an explicit expression valid within the integration interval. And one thing more: resistor $\mathrm{r}$ could also be included in an explicit linear expression like $\mathrm{I}=\mathrm{A} \cdot \mathrm{R}_{0}+\mathrm{B} . \mathrm{r}+\mathrm{C}$, again valid within the integration intervals of both r.v.

These ideas are consistent with the building of a Taylor series to represent an implicit function near the nominal values, a topic discussed by mathematicians. Such a series is an explicit representation of an implicit function. For small distances from the nominal point, the Taylor expansion may even be reduced to the linear terms only. In our case, what we are proposing could be called the "integral analog" of a Taylor series: an explicit, linear function of the random variables, good within the integration intervals.

Then, let us go on and implement the idea and see what happens.

As before, we turn to symbols $(\mathrm{x}, \mathrm{y}, \mathrm{w})$ for better interaction with computers. Variable $\mathrm{x}$ refers to resistor $\mathrm{r}$, which now comes back to be a r.v.

The first step is to find the unknown coefficients $(\mathrm{A}, \mathrm{B}, \mathrm{C})$ in the expression (the equation of a plane):

$$
\mathrm{w}=\mathrm{A} \cdot \mathrm{x}+\mathrm{B} \cdot \mathrm{y}+\mathrm{C}
$$

when $\mathrm{x}$ and $\mathrm{y}$ define three non-aligned points.

The selected points are: $(x=1.1, y=11),(x=0.9, y=9),(x=0.9, y=11)$. For each point, the corresponding value of $\mathrm{w}$ is computed by finding the root of the cubic and then selecting the right root.

We end up with a system of three linear equations in the three unknown A, B, C:

$$
\begin{aligned}
(1.1) \cdot A+(11) \cdot B+C & =0.07359 \\
(0.9) \cdot A+(9) \cdot B+C & =0.0880 \\
(0.9) \cdot A+(11) \cdot B+C & =0.07447
\end{aligned}
$$

After solving the linear system, the explicit representation of the current is (with perhaps too many decimals):

$$
\mathrm{w}=-(0.00437068) \cdot \mathrm{x}-(0.00678341) \cdot \mathrm{y}+0.15301962
$$

The next step is again calculating mean and variance by using the above expression as an explicit equation for the current, valid as a good approximation within the integration intervals:

mean of current $\mathrm{m}_{\mathrm{I}}=$

$$
\int_{0.9}^{1.1} \int_{9}^{11} 2.5(-0.00437068 x-0.00678341 y+0.15301962) d y d x=0.0808148
$$


variance of current $\sigma_{1}^{2}=$

$$
\begin{aligned}
& \int_{0.9}^{1.1} \int_{0}^{11} 2.5(-0.00437068 x-0.00678341 y+0.15301962-0.0808148)^{2} d y d x= \\
& 0.0000154019
\end{aligned}
$$

These values are compatible with those obtained by other methods and show the feasibility of integral method in cases involving polynomials.

\section{Concluding remarks}

This paper has been written to investigate why in Uncertainty Propagation the use of integrals has traditionally been superseded by derivatives, even by legal bodies like the BIPM, in spite of the fact that mean and variance of continuous random variables are defined by integrals.

An advantage of differentiation over integration may stay on the fact that differentiating is simpler than integrating. Or rather it was, up to the twentieth century. But now, in the third millennium, with great computer power at disposal, that advantage tends to be minimized.

In this study, we compared the two methods, differentiate and integrate, over various kinds of problems, to see whether integrating implies some disadvantage over differentiating beside the obvious fact of needing ... an integration! Here we summarize our findings.

In cases like linear electrical circuits, the integral method works as well as the standard BIPM method. Multiple integrals may look scaring but: a) many numeric integral calculators are at disposal from computer packages; b) the assumed independency of random variables also means that each integral does not depend on the other integrals (the case of correlated variables, although treated in the BIPM literature, was not examined here).

The differential method obviously requires that all the functions of interest be differentiable. Since integration is traditionally more tolerant of a function bad behavior than differentiation is, it seems that a method based on integration could work even when the measurand contains a step function - a non-differentiable but integrable function. To test this hypothesis, an example was created around an electric water heater whose resistor becomes disconnected from the electrical supply on insufficient water flow.

The idea to use some differentiable function to simulate a step function was examined in a separate section on sigmoids. It turns out that sigmoids have no use in differential methods but they may be helpful in integral methods.

Finally, the question whether integrals could handle implicit functions was examined and it returned interesting results. The example used in the study - a resistor whose resistance increases as it is heated by the current flowing in it - is solved by a careful numeric integration readily made in Python. It also became apparent that the implicit function 
itself may be explicitly represented by a linear expression of the random variables, good for values of the variables inside their integration limits. The expression could be considered as the integral analog of a first-order Taylor series' explicit representation of an implicit function.

The author hopes that this paper could attract others to investigate into the unjustly forgotten world of integrals in their studies of Uncertainty Propagation. Here, only specific examples are presented but a general theory is welcomed.

\section{Acknowledgment}

The author wishes to thank Dr. Denise Consonni for many helpful criticisms. 